\title{
Effects of aerobic exercise on selected physiological parameters and quality of life in patients with type 2 diabetes mellitus
}

This article was published in the following Dove Press journal:

International Journal of General Medicine

25 October 2011

Number of times this article has been viewed

\author{
Ajediran I Bello' \\ Emmanuel Owusu-Boakye' \\ Babatunde OA Adegoke ${ }^{2}$ \\ David N Adjei ${ }^{3}$ \\ 'Department of Physiotherapy, \\ School of Allied Health Sciences, \\ College of Health Sciences, Accra, \\ University of Ghana, Accra, Ghana; \\ ${ }^{2}$ Department of Physiotherapy, \\ College of Medicine, University of \\ Ibadan, Ibadan, Nigeria; ${ }^{3}$ Department \\ of Medical Laboratory Sciences, \\ School of Allied Health Sciences, \\ College of Health Sciences, \\ University of Ghana, Accra, Ghana
}

Correspondence: Ajediran I Bello Department of Physiotherapy, School of Allied Health Sciences, College of Health Sciences, University of Ghana, Accra, Ghana Tel +249294930

Fax +23321688291

Email badmus@chs.edu.gh
Background: The aim of this study was to evaluate the effects of an 8-week aerobic exercise program on physiological parameters and quality of life in patients with type 2 diabetes mellitus.

Methods: Patients attending a diabetes clinic participated in this randomized control trial. They were randomly assigned to an intervention or control group by ballot. The intervention group, in addition to regular conventional treatment, received individually prescribed aerobic exercise for 30 minutes, at $50 \%-75 \%$ of maximum heart rate three times weekly. Main outcome measures included fasting blood sugar, glycosylated hemoglobin $\left(\mathrm{HbA}_{1 \mathrm{c}}\right)$, highdensity lipoprotein, low-density lipoprotein, and a World Health Organization quality of life questionnaire (WHOQoL-BREF). Data analysis involved paired and unpaired $t$-tests and mixeddesign two-way analysis of variance.

Results: Eighteen patients with type 2 diabetes and of mean age $46.22 \pm 9.79$ years participated in the study. Mean duration since onset of diabetes in the intervention and control groups was $4.44 \pm 3.33$ years and $3.92 \pm 2.66$ years, respectively. Both groups were similar for duration since onset, baseline physiological parameters, and quality of life. Within-group comparison did not show any significant differences $(P>0.05)$ for $\mathrm{HbA}_{1 c}$, fasting blood sugar, low-density lipoprotein, or high-density lipoprotein. The intervention group improved significantly $(P<0.05)$ in their postexercise quality of life compared with baseline. Between-group comparison did not show any significant differences in physiological parameters or quality of life.

Conclusion: Patients with type 2 diabetes improved in fasting blood sugar, low-density lipoprotein, high-density lipoprotein, and quality of life following 8 weeks of aerobic exercise training. These perceived improvements were not reflected by statistically significant differences in between-group comparison for any parameters.

Keywords: type 2 diabetes mellitus, aerobic exercise, physiological parameters, quality of life

\section{Introduction}

Type 2 diabetes mellitus is closely associated with both acquired and genetic risk factors. Sedentary lifestyle is an example of an acquired factor that is an independent predictor of poor quality of life, and should be considered as a modifiable risk factor in the general population. ${ }^{1,2}$ Although there has been consensus among most researchers about the favorable effects of physical activity with regard to type 2 diabetes, studies documenting beneficial physiological adaptations in patients suffering from this disorder are not readily available in Ghana. Therefore, a pragmatic method for exercise prescription needs to be established and utilized to curb this emerging menace in regional and geographical contexts. 
The incidence of type 2 diabetes in developing countries is rising owing to a proportionate increase in many risk factors such as dietary change, increasing prevalence of obesity, and a decrease in physical activity. ${ }^{3}$ The potential efficacy of physical activity in the form of aerobic exercise is premised on modification of both insulin resistance and insulin secretion as two of the fundamental defects implicated in the pathogenesis of type 2 diabetes. ${ }^{4}$ Physical activity may slow the initiation and progression of type 2 diabetes and its cardiovascular sequelae by having favorable effects on body weight, insulin sensitivity, glycemic control, blood pressure, lipid profile, fibrinolysis, endothelial function, and inflammatory defense systems. ${ }^{5}$

A series of interventional studies has consistently supported engagement in physical activity as a way of improving glycemic control in individuals with type 2 diabetes. A meta-analysis of controlled clinical studies of the effects of exercise on glycemic control and weight in type 2 diabetes patients reported a significant reduction of $0.66 \%$ in glycosylated hemoglobin $\left(\mathrm{HbA}_{1 \mathrm{c}}\right)$ after $\geq 8$ weeks of aerobic exercise. ${ }^{6}$ Similarly, a review of prospective studies published between 1990 and 2000 concluded that reduction in the risk of type 2 diabetes associated with a physically active lifestyle is $30 \%-50 \%$ when compared with a sedentary lifestyle. ${ }^{7}$

However, in spite of the reported benefits of physical exercise, the impact of aerobic exercise on quality of life in type 2 diabetes patients remains unclear. Tessier et al reported no significant difference in quality of life between control and experimental groups following 16 weeks of treadmill exercise in elderly adults with type 2 diabetes, although no deterioration in quality of life was recorded after the training period. ${ }^{1}$ A similar study in young Finnish adults associated a higher physical fitness index with more favorable scores in general health, physical functioning, mental health, and vitality dimensions of health-related quality of life. ${ }^{8}$ The inconsistent findings surrounding this topic prompted us to undertake this research.

This study was designed to determine the effects of an 8 -week prescribed aerobic exercise program on physiological parameters and quality of life in patients with type 2 diabetes who were attending the National Diabetes Management and Research Centre at Korle-Bu Teaching Hospital, Accra, Ghana.

\section{Materials and methods Participants}

The study was approved by the ethics committee of the School of Allied Health Sciences, University of Ghana.
Participants gave their written informed consent to participate in the study. Twenty-six patients were initially contacted for the study, in anticipation of dropout during the course of the study. Eighteen participants eventually took part and completed this controlled clinical trial. They were randomly assigned to the intervention group or to the control group by ballot, without replacement in either of the groups. Participants were equally allocated to either group by allowing them to choose one option (intervention or control) from a sealed brown envelope. The allocation was conducted solely by the principal researchers. The laboratory personnel were blinded to group allocation to prevent bias during blood analysis.

\section{Inclusion criteria}

Patients were included if they met the following criteria: attendance at the National Diabetes Management and Research Centre, Korle-Bu Teaching Hospital; a diagnosis of type 2 diabetes for less than 10 years; ambulant status; age 20-65 years; and an inactive lifestyle, ie, undertaking $<20$ minutes of moderately vigorous activity, twice weekly, over the last year.

\section{Exclusion criteria}

Patients with type 2 diabetes who presented with the following were excluded from the study: a diagnosis of type 2 diabetes for more than 10 years; systolic and diastolic blood pressure more than 140 and $90 \mathrm{mmHg}$, respectively; not self-ambulatory; and bilateral or unilateral lower limb amputation.

\section{Procedure}

A venous blood sample was taken after 14 hours of fasting, with no alcohol intake for the preceding 3 days. The sample was analyzed for fasting blood sugar, $\mathrm{HbA}_{\mathrm{lc}}$, high-density lipoprotein, and low-density lipoprotein at baseline for both groups. Participants in the intervention group, in addition, were required to undergo physical examination using a maximal graded exercise tolerance test with electrocardiography at the Electrocardiography Unit, Korle-Bu Teaching Hospital. They were also screened for cardiovascular diseases according to blood pressure scores prior to exercise. Participant quality of life was also assessed at baseline using the abbreviated version of the World Health Organization quality of life questionnaire (WHOQoL-BREF).

Nine participants underwent a supervised aerobic training program lasting for 8 weeks, three times per week, with a $30-$ minute structured exercise session. Each session 
consisted of a warm-up, an aerobic phase, and cool down. Aerobic exercise intensity was individually prescribed using a bicycle ergometer but within $50 \%-75 \%$ of the maximal heart rate range for participants. ${ }^{9}$ The bicycle ergometry exercise started with the participants sitting on the bicycle with initial resistance of 10-20 watts (warm-up phase) that was gradually increased by 5-10 watts after 3 minutes (aerobic phase). The resistance was gradually reduced 5 minutes before the end of the session (cool down phase). The exercise program was conducted at the outpatient unit of the Physiotherapy Department, Korle-Bu Teaching Hospital, while they also continued their conventional care at the National Diabetes Management and Research Centre.

The control group continued with their conventional treatment at the National Diabetes Management and Research Centre, and were put on a waiting list for an aerobic exercise protocol similar to that used in the intervention group until the study was completed. Venous blood analysis and quality of life assessment were repeated after 8 weeks in both study groups.

\section{Data analysis}

Nine participants in each group whose data were consistent in terms of follow-up assessment were eventually used for analysis. Data were analyzed using SPSS version 16.0 (SPSS Inc, Chicago, IL). Descriptive statistics of means and standard deviations were used to present changes in fasting blood sugar, $\mathrm{HbA}_{\mathrm{lc}}$, high-density lipoprotein, lowdensity lipoprotein, and quality of life scores. Within-group comparisons were performed to test for differences in these parameters before and after the exercise program, while a mixed-design two-way analysis of variance was used to compare between-group differences for all the variables before and after exercise. $P<0.05$ was considered to be a statistically significant difference.

\section{Results}

The mean duration since onset of diabetes for the intervention and control groups was $4.44 \pm 3.33$ years and $3.92 \pm 2.66$ years, respectively. There was no significant difference $(P>0.05)$ in mean age and mean duration of diabetes between the intervention and control groups at baseline. Mean baseline physiological parameters of the participants in both groups were also similar, as shown in Table 1. Comparison of mean baseline and week 8 fasting blood sugar, $\mathrm{HbA}_{1 \mathrm{c}}$, high-density lipoprotein and low-density lipoprotein values did not show any significant differences $(P>0.05)$ between the groups (Table 2). Performance of the participants on the WHOQoL-BREF is shown in Figure 1. Between-group comparison of mean scores did not produce any significant differences for fasting blood sugar, $\mathrm{HbA}_{\mathrm{lc}}$, high-density lipoprotein, low-density lipoprotein, or quality of life score following the 8-week program in either group (Table 3).

\section{Discussion}

Use of physical activity in the form of aerobic exercise is widespread, with a general consensus about its beneficial effects in patients with type 2 diabetes. The therapeutic benefits include regulation of body weight, reduction of insulin resistance, enhancement of insulin sensitivity, and glycemic control. However, despite its importance, the mode of prescription remains a matter of debate. The aim of this study was to investigate the effects of an 8-week aerobic exercise program on fasting blood sugar, $\mathrm{HbA}_{1 \mathrm{c}}$, high-density lipoprotein, low-density lipoprotein, and quality of life in patients with type 2 diabetes. Clinical evaluation of the participants who engaged in the 8-week program showed some beneficial effects when combined with conventional routine care on metabolic parameters, as well as on quality of life in the participants.

Table I Physical and clinical characteristics of the participants

\begin{tabular}{|c|c|c|c|c|}
\hline Variables & $\begin{array}{l}\text { Intervention } \\
(\mathrm{n}=9)\end{array}$ & $\begin{array}{l}\text { Control } \\
(n=9)\end{array}$ & t-test & $P$ value \\
\hline Mean age \pm SD (years) & $43.33 \pm 6.73$ & $45.11 \pm 11.82$ & -1.28 & 0.22 \\
\hline Mean diabetes duration \pm SD (years) & $4.44 \pm 3.33$ & $3.92 \pm 2.66$ & 0.36 & 0.73 \\
\hline Mean $\mathrm{BMI} \pm \mathrm{SD} \mathrm{kg} / \mathrm{m}^{2}$ & $28.30 \pm 4.24$ & $26.12 \pm 3.51$ & 1.19 & 0.25 \\
\hline Mean baseline FBS $\pm \mathrm{SD}(\mathrm{mmol} / \mathrm{l})$ & $9.26 \pm 1.90$ & $9.58 \pm 4.4 \mathrm{I}$ & -0.20 & 0.85 \\
\hline Mean baseline $\mathrm{HbA}_{\mathrm{Ic}} \pm \mathrm{SD}(\%)$ & $8.20 \pm 1.74$ & $7.91 \pm 2.47$ & 0.29 & 0.78 \\
\hline Mean baseline HDL \pm SD (mmol/L) & $1.33 \pm 0.37$ & $1.52 \pm 0.37$ & -1.07 & 0.30 \\
\hline Mean baseline LDL \pm SD (mmol/L) & $2.84 \pm 0.48$ & $2.51 \pm 0.55$ & 1.37 & 0.19 \\
\hline Mean baseline QOL \pm SD (\%) & $61.07 \pm 9.00$ & $58.37 \pm 15.43$ & 0.46 & 0.66 \\
\hline
\end{tabular}

Abbreviations: QOL, quality of life; FBS, fasting blood sugar; HbA deviation; BMI, body mass index. 
Table 2 Paired $t$-test comparison between physiological parameters and quality of life scores within groups at baseline and week 8

\begin{tabular}{|c|c|c|}
\hline Outcomes & Intervention $(\mathrm{n}=9)$ & Control $(n=9)$ \\
\hline \multicolumn{3}{|l|}{ Fasting blood sugar (mmol/L) } \\
\hline Baseline, week $8, t$ value, $P$ value & $9.26 \pm 1.90,8.91 \pm 2.01,-0.06,0.96$ & $9.58 \pm 4.4 \mathrm{I}, 9.10 \pm 3.40,-0.1 \mathrm{I}, 0.92$ \\
\hline \multicolumn{3}{|l|}{ Glycosylated Hemoglobin (\%) } \\
\hline Baseline, week $8, t$ value, $P$ value & $8.20 \pm 1.74,8.38 \pm 1.74,-2.91,0.65$ & $7.91 \pm 2.47,7.73 \pm 2.12,0.48,0.65$ \\
\hline \multicolumn{3}{|c|}{ High-density lipoproteins (mmol/L) } \\
\hline Baseline, week $8, t$ value, $P$ value & $\mathrm{I} .33 \pm 0.37, \mathrm{I} .45 \pm 0.48,-0.47,0.35$ & $1.52 \pm 0.37,1.60 \pm 0.47,-0.83,0.44$ \\
\hline \multicolumn{3}{|c|}{ Low-density lipoproteins (mmol/L) } \\
\hline Baseline, week $8, t$ value, $P$ value & $2.84 \pm 0.48,2.81 \pm 0.78,0.18,0.87$ & $2.51 \pm 0.55,2.40 \pm 0.55,0.30,0.78$ \\
\hline \multicolumn{3}{|l|}{ Quality of life (\%) } \\
\hline Baseline, week $8, t$ value, $P$ value & $61.07 \pm 9.00,65.28 \pm 13.03,-0.06,0.03$ & $58.37 \pm 15.43,55.53 \pm|5.05,-0.7|, 0.20$ \\
\hline
\end{tabular}

Note: $P<0.05$.

Comparison of the baseline and week 8 mean scores for the physiological variables in each group showed improvement over baseline, but the differences were not statistically significant. This implies that patients with type 2 diabetes are more likely to benefit from an 8-week aerobic exercise program in addition to their conventional care. This finding follows the same trend as that of a recent meta-analysis of studies that utilized 2-12 months of exercise in subjects with type 2 diabetes and found no significant changes in high-density lipoprotein or low-density lipoprotein. ${ }^{10} \mathrm{~A}$ recent 9-month study in young healthy adults showed only an insignificant trend toward low-density lipoprotein reduction. ${ }^{11}$

Between-group comparison did not yield any significant differences in any of the parameters following the 8-week exercise program. In contrast, some previous studies have shown significant improvement in $\mathrm{HbA}_{1 \mathrm{c}}$, total cholesterol, high-density lipoprotein, low-density lipoprotein, and triglycerides in patients with type 2 diabetes who engaged in structured physical activities compared with controls, ${ }^{6,10-12}$ thereby lending credence to the general belief that aerobic exercise is beneficial in the management of patients with type 2 diabetes. The inconsistencies in these studies could be attributed to varying factors ranging from differences

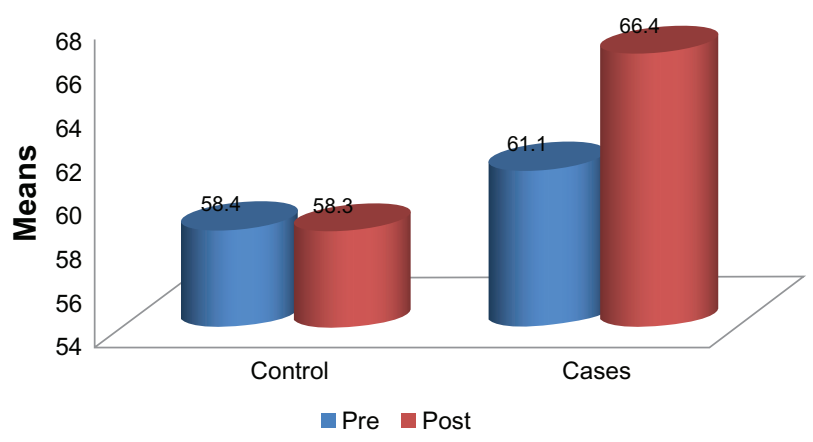

Figure I Pre-exercise and postexercise comparison of quality of life among the participants following the 8-week aerobic training. in study design to socioeconomic and sociodemographic factors. The interplay of these factors might have influenced the outcomes of the previous and present studies. There is a need to replicate research in this environment, perhaps for a longer duration in order to confirm the present findings.

Although a significant difference was found before and after the exercise program for quality of life in favor of the intervention group, there was no significant difference between the two groups in the present study. This outcome supports the findings of previous investigators who reported no significant change in quality of life scores between control and experimental groups following 16 weeks of treadmill exercise in elderly adults with type 2 diabetes, although no deterioration in quality of life was recorded after the training period. ${ }^{1}$ Another study in young Finnish adults associated a higher physical fitness index with more favorable scores in general health, physical functioning, mental health, and vitality dimensions of health-related quality of life. ${ }^{8}$

Table 3 Between groups comparison of the selected parameters using two-way analysis of variance

\begin{tabular}{llll}
\hline Parameters & Mean square & F value & P value \\
\hline FBS & & & \\
Pre-exercise & 0.432 & 0.039 & 0.846 \\
$\begin{array}{l}\text { Postexercise } \\
\text { LDL }\end{array}$ & 0.019 & 0.013 & 0.910 \\
$\begin{array}{l}\text { Pre-exercise } \\
\text { Postexercise }\end{array}$ & 0.500 & 0.500 & 0.190 \\
HbA & 0.601 & 0.601 & 0.272 \\
Pre-exercise & 0.376 & & \\
Postexercise & 1.385 & 0.376 & 0.778 \\
HDL & & 1.385 & 0.561 \\
Pre-exercise & 0.161 & & \\
Postexercise & 0.084 & 0.161 & 0.300 \\
QoL & & 0.084 & 0.551 \\
Pre-exercise & 29.3 & 29.3 & \\
Postexercise & 243.0 & 243.0 & 0.655 \\
\hline
\end{tabular}

Abbreviations: QoL, quality of life; HDL, high-density lipoprotein; LDL, lowdensity lipoprotein; $\mathrm{FBS}$, fasting blood sugar; $\mathrm{HbA}_{\mathrm{lc}}$, glycosylated hemoglobin. 
This implies that engagement in an 8-week exercise program could influence quality of life in patients with type 2 diabetes.

In conclusion, although 8-week aerobic exercise program in addition to conventional cares of patients with type-2 diabetes mellitus did not produce any significant improvement on FBS, HDL, LDL and QoL over those recieving conventional cares only, its inclusion may be beneficial on longer duration. The outcomes of this study suggest inclusion of an aerobic exercise program into the routine management of patients with type 2 diabetes could be beneficial.

\section{Acknowledgments}

We acknowledge Professor ABG Amoah who allowed us to recruit patients for the study from the National Diabetes Management and Research Centre, Korle-Bu Teaching Hospital, Accra, Ghana. We also acknowledge Richard K Baan and Evan Chinebuah for their assistance with blood analysis in this study.

\section{Disclosure}

The authors are not aware of any conflicts of interest in this work.

\section{References}

1. Tessier D, Menard J, Fulop T, et al. Effects of physical fitness in the elderly with type-2 diabetes mellitus. Arch Gerontol Geriatr. 2000;31: 121-132.
2. Holton DR, Colberg SR, Nunnold T, Parson HK, Vinik AI. The effect of an aerobic exercise training program on quality of life in type 2 diabetes. Diabetes Educ. 2003;29:837-846.

3. Soria MLB, Sy RG, Vega BS, Ty-Willing T, Abenir-Gallardo A, Vellandria F, Punzallan FE. The incidence of type 2 Mellitus in Phillipines: A 9-year cohort study. Diabetes Res and Clin Prac. 2009; 86:130-133

4. Nagi D, editor. Exercise and Sport in Diabetes. 2nd ed. New York, NY: John Wiley \& Sons Ltd; 2005.

5. Bassuk SS, Manson JE. Epidemiological evidence for the role of physical activity in reducing risk of type 2 diabetes and cardiovascular disease. J Appl Physiol. 2005;99:1193-1204.

6. Boulé NG, Haddad E, Kenny GP, Wells GA, Sigal RJ. Effects of exercise on glycemic control and body mass in type 2 diabetes mellitus: a metaanalysis of controlled clinical trials. JAMA. 2001;286:1218-1227.

7. Skerrett PJ, Manson JE. Reduction in risk of coronary heart disease and diabetes. In: Ruderman N, Devlin JT, Schneider SH, Kriska A, editors. Handbook of Exercise in Diabetes. Alexandria, VA: American Diabetic Association; 2002.

8. Hakkinen A, Rinne M, Vansakari T, Santilla M, Hakkinen K, Kyrolainen. Association of physical fitness with health related quality of life in Finnish young men. Available at: http://www.Hqol.com/content/8/1/15. Accessed April 26, 2011.

9. Gormley J, Hussey J, editors. Exercise Therapy, Prevention and Treatment of Disease. Oxford, UK: Blackwell Publishers Ltd; 2005.

10. Thomas DE, Elliott EJ, Naughton GA. Exercise for type 2 diabetes mellitus. Cochrane Database Syst Rev. 2006;3:CD002968.

11. Ring-Dimitriou S, von Duvillard SP, Paulweber B, et al. Nine months aerobic fitness induced changes on blood lipids and lipoproteins in untrained subjects versus controls. Eur J Appl Physiol. 2007;99: 291-299.

12. Van Rooijen AJ, Viviers CM, Becker PJ.A daily physical activity and diet intervention for individuals with type 2 diabetes mellitus: A randomized controlled clinical trial. South Afr J Physiother. 2010;66:9-15.
International Journal of General Medicine

\section{Publish your work in this journal}

The International Journal of General Medicine is an international, peer-reviewed open-access journal that focuses on general and internal medicine, pathogenesis, epidemiology, diagnosis, monitoring and treatment protocols. The journal is characterized by the rapid reporting of reviews, original research and clinical studies across all disease areas.

\section{Dovepress}

A key focus is the elucidation of disease processes and management protocols resulting in improved outcomes for the patient.The manuscript management system is completely online and includes a very quick and fair peer-review system. Visit http://www.dovepress.com/ testimonials.php to read real quotes from published authors. 